\title{
Aeroacústica de emparelhamento de vórtices em esteiras assimétricas
}

\author{
Marlon S. Mathias, \\ Departamento de Engenharia Aeronáutica, EESC, USP, \\ 13563-120, São Carlos, SP \\ E-mail: marlon.mathias@usp.br, \\ Elmer M. Gennaro \\ Unesp - Campus de São João da Boa Vista \\ São João da Boa Vista, SP \\ E-mail: elmer.gennaro@sjbv.unesp.br,

\section{Marcello A. F. Medeiros} \\ Departamento de Engenharia Aeronáutica, EESC, USP, \\ 13563-120, São Carlos, SP \\ E-mail: marcello@sc.usp.br.
}

\begin{abstract}
Resumo: O objetivo deste estudo é analisar a aeroacústica de esteiras assimétricas. Tal escoamento pode ser encontrados a jusante de perfis aerodinâmicos que geram sustentação, dispositivos hipersustentadores de aeronaves ou de tubos próximos a paredes, por exemplo. A evolução do escoamento é calculada por simulação numérica direta, na qual são induzidas perturbações controladas, podendo-se obter o comportamento aeroacústico para diferentes frequências. São formados vórtices assimétricos que se emparelham. Tanto a formação quanto o emparelhamento geram som. Devido à assimetria, este som se propaga de maneira distinta em diferentes direções. Estudando-se os fatores que influenciam nesta distribuição pode-se entender melhor a aeroacústica de corpos de formas mais complexas. Futuramente tal conhecimento pode ser aplicado na concepção de aeronaves mais silenciosas, cumprindo requisitos ambientais mais rígidos.
\end{abstract}

Palavras-chave: Aeroacústica, DNS, Escoamento Cisalhante, Esteira Assimétrica

\section{Introdução}

Durante procedimentos de pouso ou decolagem uma aeronave produz um alto nível de ruído. Tem havido um crescimento nas exigências ambientais nas proximidades de aeroportos. Com o avanço na tecnologia de motores mais silenciosos, os dispositivos hipersustentadores passaram a ter uma posição de destaque entre as fontes sonoras, em especial os slats durante o pouso.

Escoamentos viscosos ao redor de corpos rombudos geram uma esteira; onde, em uma certa faixa de número de Reynolds, passa a ocorrer um desprendimento de vórtices devido à instabilidade. Em corpos que geram sustentação, à esteira é somada uma componente assimétrica. Esse é o caso de slats, flaps e outros dispositivos aerodinâmicos.

Em uma esteira simétrica o sistema de vórtices gera relativamente pouco som. Nestes casos a geração sonora está associada à força oscilatória sobre o corpo. Isto se deve ao fato de que o arranjo de vórtices em uma esteira simétrica é muito estável não ocorrendo grande deformação nos vórtices, o que é um mecanismo importante de geração de som.

No caso assimétrico, esses vórtices possuem intensidades diferentes, e a instabilidade que se verifica no caso simétrico é perdida. No arranjo assimétrico ocorrem grandes deformações neste 
vórtices e a consequente geração de som pode ser relevante. Em algumas circunstâncias ocorre emparelhamento de vórtices, o que é uma importante fonte sonora. A assimetria da esteira pode, portanto, alterar as características do som gerado, tanto em sua intensidade quanto na direção de sua propagação.

Esteiras assimétricas podem ser encontradas, também, em outras situações. Um cilindro imerso em um escoamento cisalhante, exemplificado por um tubo ou cabo dentro da camada limite de uma parede, é uma outra situação que gera tal estrutura.

Entre os efeitos da assimetria está, também, uma acentuação ou atenuação da instabilidade da esteira, o que influencia o som gerado.

A aeroacústica de uma camada de mistura foi estudada por[2] e [3]. Os efeitos da assimetria numa esteira sobre a formação de vórtices já foram estudados por diversos autores nem sempre chegando às mesmas conclusões como observou [5].

O objetivo deste trabalho é entender a influência de cada parâmetro na instabilidade e no som gerado. Incluindo o número de Reynolds, de Mach, a frequência da oscilação, a assimetria. As ferramentas para análises de outros parâmetros também serão desenvolvidas.

\section{Metodologia}

Para o objetivo deste trabalho são usadas ferramentas computacionais tanto para simular quanto para analisar o escoamento.

Inicialmente utilizou-se, com pequenas modificações, o código DNS em Fortran desenvolvido e utilizado por um grupo de pesquisa do Departamento de Engenharia Aeronáutica da EESCUSP. Tal código é escrito em Fortran e é descrito por [2].

Concluídos os estudos preliminares, foi escrito um novo DNS em Matlab especificamente para o projeto, tanto com o objetivo de se ter um código otimizado para o problema quanto para um melhor entendimento e maior controle sobre as soluções numéricas. Certas técnicas de programação foram aplicadas para que o código em Matlab atingisse um desempenho equivalente ao código em Fortran.

A primeira parte dos trabalhos foi dedicada ao estudo do fenômeno em um domínio periódico na direção do escoamento, mais simples. Depois passou-se a um domínio aberto, mais caro e complexo, porém mais fiel à realidade. Este segundo método é detalhado a seguir.

O código usa as equações de Navier-Stokes bidimensionais compressíveis que são resolvidas numericamente por um DNS, Direct Numeric Solver, isto é, sem modelos de turbulência.

Todos os valores são adimensionais e dados em função de parâmetros adimensionais como os números de Reynolds, Mach e Prandtl. A adimensionalização possibilita o uso dos resultados em problemas de várias naturezas e dimensões.

\subsection{Adimensionalização}

Como unidade de comprimento foi escolhida a largura da esteira média entre os dois lados da esteira. A velocidade é normalizada à média do escoamento livre. A densidade inicial da fluido, sua massa molar e capacidade térmica a volume constante também são unitários.

A viscosidade, $\mu$, temperatura base, $T_{0}$, e condutividade térmica, $k$, são escolhidas para atender aos números de Reynolds, Re, de Mach, $M a$, e de Prandtl, Pr, respectivamente, por meio das equações

$$
\begin{gathered}
\mu=\frac{\rho_{0}}{R e} \\
T_{0}=\frac{1}{R \gamma M a^{2}} \\
k=\frac{C_{v} \gamma \mu}{P r}
\end{gathered}
$$




\subsection{Malha}

O código utiliza uma malha bidimensional retangular estruturada. A malha é uniforme em $x$ até o início da zone de amortecimento, onde passa a haver um estiramento no sentido de afastar os pontos. Há um estiramento em y para concentrar pontos na região de maior cisalhamento.

A dimensão do domínio em $x$ deve ser grande o suficiente para que os vórtices se desenvolvam suficientemente para o estudo antes de atingirem a zona de amortecimento, cujo funcionamento será mostrado posteriormente.

A dimensão em y é escolhida de forma que o domínio seja largo o suficiente para incluir uma região acústica e para que a solução seja independente deste domínio.

O estiramento na direção $y$ permite que mais pontos estejam na região cisalhante, onde se necessita um maior refinamento devido aos pequenos comprimentos de onda presentes, sem aumentar o número total de pontos e, consequentemente, o custo computacional. Para que as distâncias de um nó para o próximo aumentem de maneira uniforme ao se afastar do centro, foi usada a seguinte equação para se obter valores de $y$ para a malha

$$
y_{i}=\frac{L_{y} \sinh \left(A\left(\frac{2 i}{n_{y}-1}\right)-1\right)}{2 \sinh (A)} \quad i=0, \ldots, n_{y}-1
$$

A malha também contém regiões de amortecimento, usadas para evitar a reflexão das ondas. Nesta zona é somado um termo de amortecimento ao escoamento, que o faz tender ao estado inicial. É criada uma função de amortecimento $\sigma(\xi)$, mostrada na equação

$$
\sigma(\xi)=I(1-\cos (\xi))^{E} \quad 0 \leq \xi \leq \frac{\pi}{2}
$$

Esta equação é transformada linearmente para cada zona de amortecimento, com $\xi=0$ em seu início e $\xi=\pi / 2$ no fim do domínio.

\subsection{Estrutura de dados}

Como uma malha estruturada é usada, todos os dados podem ser armazenados na forma de matrizes, onde cada linha representa os nós com uma coordenada y fixa e cada coluna, os nós com uma coordenada $x$ fixa.

É usada uma matriz $Y_{i}$ para cada variável calculada. Para esta formulação, são quatro matrizes de tamanho $n_{y} \times n_{x}$, sendo este, também, o tamanho da malha.

Matrizes esparsas com os coeficientes para as derivadas espaciais e para o filtro numérico são geradas e armazenadas ao início da execução. Estas serão discutidas nas seções seguintes.

Esta organização foi escolhida pois permite que apenas operações matriciais sejam usadas durante a execução, permitindo tirar vantagem do alto desempenho do Matlab com matrizes e de sua paralelização embutida.

\subsection{Esquemas numéricos}

Por se tratar de um DNS, as equações de Navier-Stokes são usadas diretamente, sem modelagem de turbulência. O sistema de equações é mostrado a seguir, como descrito por [1], mas considerando-se apenas duas dimensões.

$$
\mathbf{U}=\left\{\begin{array}{l}
\rho \\
\rho u \\
\rho v \\
\rho\left(e+\frac{V^{2}}{2}\right)
\end{array}\right\}
$$




$$
\begin{aligned}
\mathbf{F}=\left\{\begin{array}{l}
\rho u \\
\rho u^{2}+p-\tau_{x x} \\
\rho v u-\tau_{x y} \\
\rho\left(e+\frac{V^{2}}{2}\right) u+p u-k \frac{\partial T}{\partial x}-u \tau_{x x}-v \tau_{x y}
\end{array}\right\} \\
\mathbf{G}=\left\{\begin{array}{l}
\rho v \\
\rho u v-\tau_{x y} \\
\rho v^{2}+p-\tau_{y y} \\
\rho\left(e+\frac{V^{2}}{2}\right) v+p v-k \frac{\partial T}{\partial y}-u \tau_{x y}-v \tau_{y y}
\end{array}\right\} \\
\frac{\partial \mathbf{U}}{\partial t}=-\frac{\partial \mathbf{F}}{\partial x}-\frac{\partial \mathbf{G}}{\partial y}
\end{aligned}
$$

As derivadas espaciais são obtidas por um esquema de diferenças finitas explícito de quarta ordem. Para se tirar vantagem das operações matriciais otimizadas do Matlab, os coeficientes das diferenças finitas foram colocados em matrizes da seguinte forma

$$
\mathbf{D}_{\xi}=\left[\begin{array}{ccccccc}
0 & a_{1} & a_{2} & & & -a_{2} & -a_{1} \\
-a_{1} & 0 & a_{1} & a_{2} & & & -a_{2} \\
\ddots & \ddots & \ddots & \ddots & \ddots & & \\
& -a_{2} & -a_{1} & 0 & a_{1} & a_{2} & \\
& & \ddots & \ddots & \ddots & \ddots & \ddots \\
a_{2} & & & -a_{2} & -a_{1} & 0 & a_{1} \\
a_{1} & a_{2} & & & -a_{2} & -a_{1} & 0
\end{array}\right]_{\left[n_{y} \times n_{y}\right]}
$$

Onde $a_{1}=2 / 3$ e $a_{2}=-1 / 12$. Para se obter as derivadas, basta multiplicar essa matriz $D_{\xi}$ por uma matriz $Y$ qualquer contendo as variáveis ao longo de uma malha de espaçamento unitário. Como a malha usada não tem espaçamento unitário nem uniforme, é usada a regra da cadeia. Para a direção $y$, por exemplo,

$$
\mathbf{D}_{\mathbf{y}}^{*}=\left(\mathbf{I}_{\mathbf{n}_{\mathbf{y}}}\left(\mathbf{D}_{\xi} \mathbf{y}\right)\right)^{-1} \mathbf{D}_{\xi}
$$

A matriz $D_{y}^{*}$ já considera o espaçamento e o estiramento da malha corretamente, mas assume um domínio periódico, note que pontos no em um lado do domínio influenciam as derivadas de pontos no outro lado. Para se considerar o contorno, os coeficientes das duas primeiras e duas últimas linhas são substituídos pelos obtidos a partir de polinômios de Lagrange, criando derivadas descentradas nessas regiões. Obtém-se, assim, a matriz $D_{y}$ para derivadas em $y$. A matriz $D_{x}$ para derivadas em $x$ é obtida de maneira análoga, mas substituindo-se as primeiras e últimas colunas. A fim de se reduzir o custo computacional, essas matrizes são alocadas na memória como esparsas e computadas apenas uma vez, já que se mantém constantes durante a simulação.

Ao se multiplicar $D_{x}$ ou $D_{y}$ por $Y$ obtemos as derivadas em $x$ e em $y$ respectivamente. Note que $D_{x}$ deve ser o segundo termo da multiplicação e $D_{y}$ deve ser o primeiro. Isso se deve ao fato de a derivada em $x$ operar em cada linha da matriz separadamente enquanto a derivada em $y$ opera em cada coluna.

A evolução temporal é feita pelo método de Runge-Kutta de quarta ordem, a equação final do método é modificada como mostrado a seguir

$$
\mathbf{Y}^{\mathbf{n}+\mathbf{1}}=\mathbf{Y}^{\mathbf{n}}+\frac{d t}{6}\left(\mathbf{K}_{\mathbf{1}}+2 \mathbf{K}_{\mathbf{2}}+2 \mathbf{K}_{\mathbf{3}}+\mathbf{K}_{\mathbf{4}}\right)-d t\left[\sigma\left(Y^{n}-Y^{0}\right)\right]_{(i, j)}
$$

O último termo da equação se refere à zona de amortecimento. $\sigma$ vale zero em quase todo o domínio exceto em regiões próximas ao contorno de acordo com a Equação 5. Isso é feito de modo a evitar que as ondas reflitam na borda e voltem ao centro do domínio. 
Em alguns casos também se usou uma separação de fluxos na zona de amortecimento, como mostrado por [7] e [8], cancelando-se os fluxos de entrada na região de amortecimento.

O passo de tempo a ser tomado é definido pela condição de estabilidade CFL, como feito por [6] e segue as equações a seguir, de modo que $C F L<1$

$$
\begin{gathered}
d t \leq \frac{C F L}{\left(\frac{\frac{1}{M}+u_{\max }}{d x_{\min }}+\frac{\frac{1}{M}+v_{\max }}{d y_{\min }}\right)} \\
d t \leq \frac{R e}{\left(\frac{1}{d x_{\min }^{2}}+\frac{1}{d y_{\min }^{2}}\right)}
\end{gathered}
$$

Após cada passo temporal é feita uma filtragem numérica para evitar o surgimento de ondas de altas frequência sem sentido físico causadas pelo ruído numérico, como mostrado por [4]. Essa filtragem envolve resolver, para o domínio todo, um sistema linear da forma

$$
\alpha \overline{u_{i-1}}+\overline{u_{i}}+\alpha \overline{u_{i+1}}=a_{0} u i+\sum_{j=1}^{4}\left(a_{j} \frac{u_{i-j}+u_{i+j}}{2}\right)
$$

Onde $u_{i}$ denota a variável não filtrada em um ponto $i$ e $\overline{u_{i}}$ a denota após filtrada. Esse sistema é resolvido de forma matricial. A filtragem deve ser feita duas vezes, uma em cada direção. Novamente, assim como nas matrizes das derivadas, os coeficientes próximos aos contornos são devidamente alterados.

\subsection{Condição inicial}

A condição inicial do código inclui a esteira assimétrica, mas nenhuma turbulência. A esteira é modelada como a soma de uma gaussiana com uma tangente hiperbólica, como mostra a equação

$$
u(y)=A\left(1-e^{-B y^{2}}\right)+C \tanh (D y)
$$

$C$ é usado como o parâmetro de assimetria da esteira, $B$ e $D$ se relacionam à sua largura e $A$, ao déficit de velocidade da esteira.

O déficit é mantido constante e igual a $1 . B$ e $D$ são escolhidos de forma que a largura da esteira seja unitária, de acordo com o processo de adimensionalização usado.

A largura é medida como a distância entre a posição onde a velocidade é metade da do escoamento livre em cada lado da esteira, como feito por [5].

Para induzir a formação dos vórtices, é colocada uma pequena perturbação na velocidade vertical do escoamento, $v$, na entrada do domínio da forma mostrada na equação

$$
v(x=0, y, t)=e^{-E_{1} y^{2}} U_{1} \sin \left(\frac{\pi t}{\alpha}\right)+e^{-E_{2} y^{2}} U_{2} \sin \left(\frac{2 \pi t}{\alpha}\right)
$$

Duas senoides de diferentes frequências são colocadas no domínio como harmônico e subharmônico. $L_{x}$ é o período do domínio, o coeficiente $E$ controla a região do domínio perturbada e $U$ controla a intensidade da perturbação. As senoides são multiplicadas por gaussianas em y para que a perturbação se concentre na região onde há o cisalhamento, longe das condições de contorno. Esta perturbação é somada à condição de entrada do domínio.

Outra opção de perturbação usada é um ruído branco, que age em todo o espectro, não apenas nas frequências escolhidas para a perturbação. 


\section{Resultados}

As simulações foram realizadas para os seguintes valores adimensionais: número de Reynolds $R e=1000$, número de Mach $M a=0.4$ e número de Prandtl $\operatorname{Pr}=0.8$. A assimetria variou entre $0 \leq C \leq 0.3$. A malha tem resolução $200 \times 500$, com a qual foi observada a convergência dos resultados.

A velocidade de execução ficou na ordem de $1 \mu s$ por ponto por passo de tempo em um computador pessoal atual. Comparável a códigos semelhantes escritos em outras linguagens. Uma execução completa pode ser terminada em menos de um dia.

Foi observado que com o aumento da assimetria, o escoamento se torna mais complexo e passa a se assemelhar a um escoamento cisalhante, podendo até conter emparelhamentos de vórtice. A Figura 1(a) mostra a vorticidade para escoamentos com 0\%, $10 \%$ e 30\% de assimetria. Já a Figura 1(b) mostra o escoamento com $20 \%$ de assimetria, ao centro, e a pressão acústica instantânea acima e abaixo.
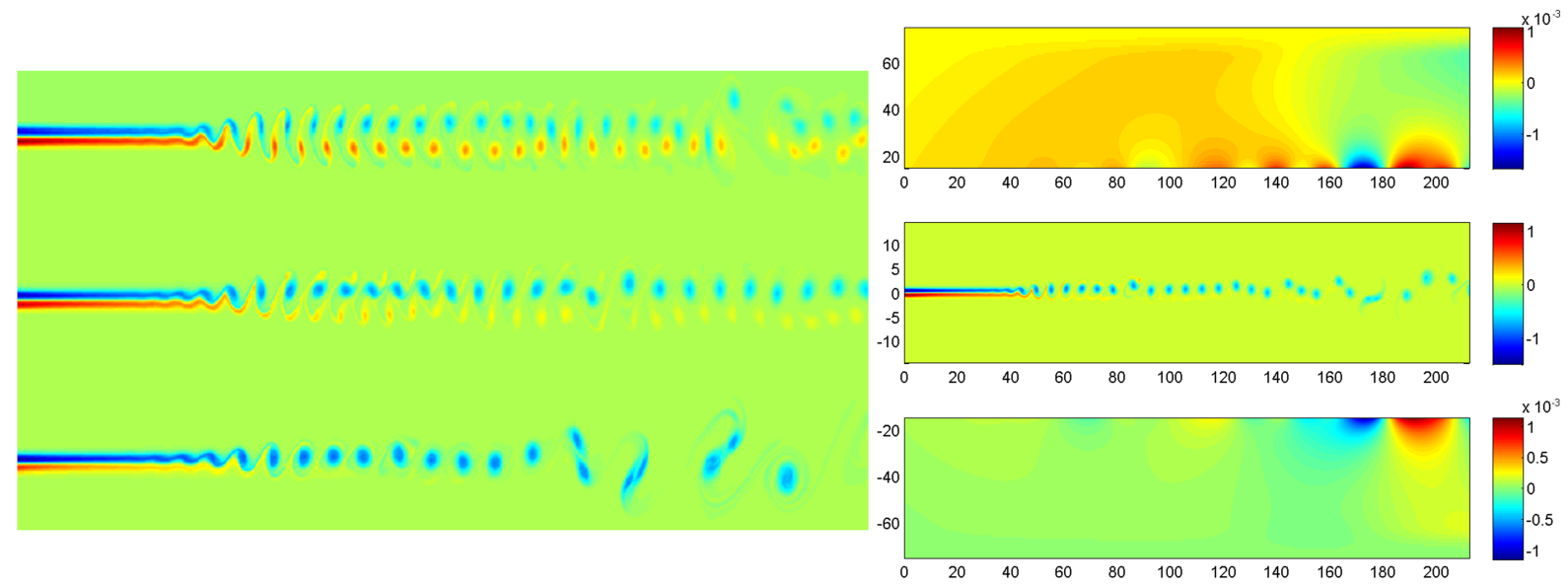

Figura 1: (a)Vorticidade para escoamentos com 0\%, $10 \%$ e 30\% de assimetria (b)Escoamento com $20 \%$ de assimetria e sua emissão acústica

Note como a assimetria influencia a forma e o tamanho dos vórtices formados, o que altera o som gerado pela esteira. Medições da pressão acústica foram feitas em linhas horizontais próximas às bordas do domínio. Para o casos simétrico e de $10 \%$ de assimetria, a emissão sonora foi relativamente pequena, mas aumento consideravelmente com $20 \%$ de assimetria e ainda mais com $30 \%$.

A Figura 2(a) mostra a pressão acústica média para diferentes posições do domínio. A Figura 2(b) mostra a densidade espectral do som em um ponto próximo ao fim do domínio.
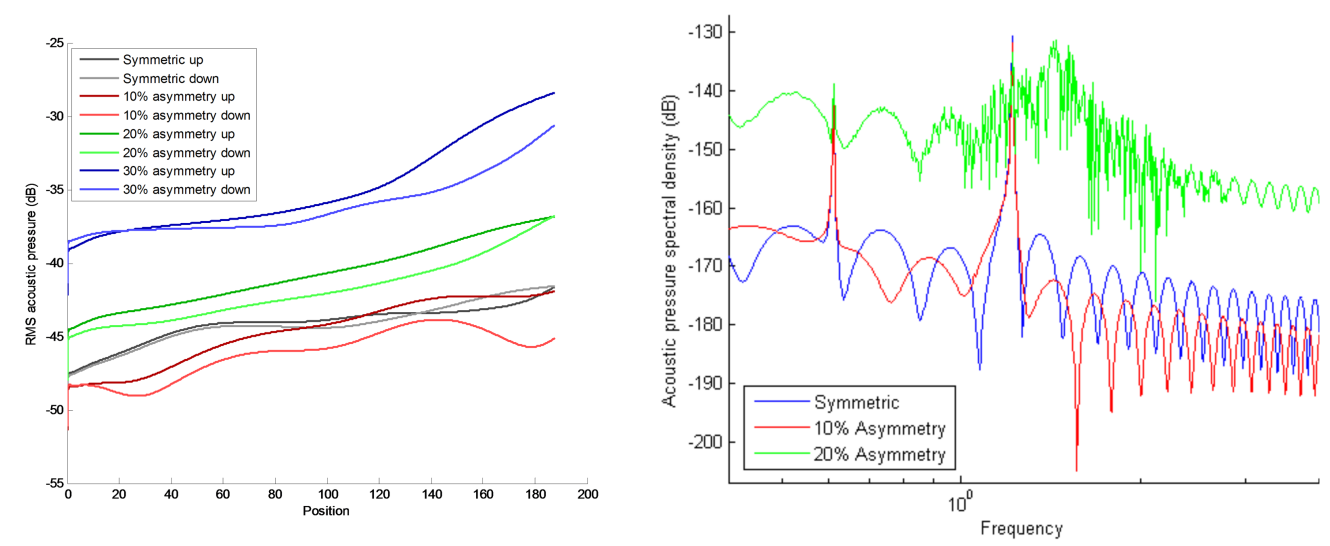

Figura 2: (a)RMS da pressão acústica em diferentes posições (b)Densidade espectral do som 
Com a maior assimetria, os vórtices se deformando e emparelhando se tornaram a fonte sonora dominante, movendo as região com maior intensidade sonora a jusante.

Nos casos simétrico e com $10 \%$ de assimetria, há dois picos claros a 0.61 e 1.22 da frequência adimensional. Com $20 \%$ de assimetria, esses picos continuam presentes, em uma magnitude semelhante, mas houve um grande aumento da emissão em outras frequências, indicando a complexidade do escoamento.

\section{Conclusão}

Foi mostrado que esteiras assimétricas compartilham características tanto com esteiras simétricas quanto com escoamentos cisalhantes. O valor de $20 \%$ de assimetria é, de modo aproximado, um divisor entre esses dois tipos de fenômeno. Esteiras com maior assimetria passam a se assemelhar mais a escoamentos cisalhantes e a emitir mais som.

Também foi mostrado ser possível desenvolver códigos computacionais DNS capazes de boa velocidade em Matlab com o uso de certas técnicas de programação, apesar de se acreditar que isso seja válido apenas para problemas bidimensionais de menor escala devido ao uso de memória.

\section{Agradecimentos}

Os autores gostariam de agradecer a todos professores e alunos que apoiaram o projeto e à Fapesp, Fundação de Amparo à Pesquisa do Estado de São Paulo, pelo apoio financeiro.

\section{Referências}

[1] J.D. Anderson Jr., "Computational Fluid Dynamics: The Basics with Applications", McGraw-Hill, 1995.

[2] A.K. Colaciti, J. Sesterhenn, R. A. C. Germanos, e M. A. F. Medeiros. "Development of a Code for Simulation of Aeroacoustic Phenomena in a Mixing Layer.", Congresso Brasileiro de Engenharia Mecânica, 2007.

[3] T. Colonious, e S. K. Lele. "Computational Aeroacustics: Progress os Nonlinear Problems of Sound Generation.", Progress in Aerospace Sciences, v.40, 2004.

[4] D.V. Gaitonde, e M.R. Visbal. "High-Order Schemes for Navier-Stokes Equations: Algorithm and Implementation.", Air Vehicles Directorate. Air Force Research Laboratory. Wright-Patterson Air Force Base, Ohio, 1998.

[5] E.M. Gennaro. "Análise da Instabilidade Hidrodinâmica de uma Esteira Assimétrica.", Dissertação de Mestrado, EESC-USP, 2008.

[6] R.A.C. Germanos. "Simulação Numérica da Evolução Linear e Não-Linear em uma Camada de Mistura Compressível Tridiensional", Tese de Doutorado, EESC-USP, 2009.

[7] J.L. Steger, R.F. Warming, "Flux Vector Splitting of the Inviscid Gasdynamic Equations with Application to Finite-Difference Methods.", Journal of Computational Physics, 1981.

[8] F.M. White, "Viscous Fluid Flow", McGraw-Hill, 1974. 